\title{
The association between omentin and diabetes: a systematic review and meta-analysis of observational studies
}

This article was published in the following Dove Press journal: Diabetes, Metabolic Syndrome and Obesity: Targets and Therapy

\author{
Atefeh As'habi ${ }^{1,2}$ \\ Malihe Sadeghi' ${ }^{3,4}$ \\ Arman Arab iD ${ }^{5}$ \\ Hossein Hajianfar ${ }^{1,2}$ \\ 'Food Safety Research Center (SALT), \\ Semnan University of Medical Sciences, \\ Semnan, Iran; ${ }^{2}$ Department of Nutrition, \\ School of Nutrition and Food Sciences, \\ Semnan University of Medical Sciences, \\ Semnan, Iran; ${ }^{3}$ Health Information \\ Technology Department, Faculty of \\ Paramedics, Semnan University of \\ Medical Sciences, Semnan, Iran; ${ }^{4}$ Health \\ Information Management, Iran University \\ of Medical Sciences, Tehran, Iran; \\ ${ }^{5}$ Department of Community Nutrition, \\ School of Nutrition and Food Science, \\ Food Security Research Center, Isfahan \\ University of Medical Sciences, Isfahan, \\ Iran
}

\begin{abstract}
Aims: A number of studies have examined the association between the serum levels of omentin and diabetes, but the findings have been inconclusive. Herein, we systematically reviewed available observational studies to elucidate the overall relationship between omentin and diabetes, including type 1 diabetes mellitus (T1DM), type 2 diabetes mellitus (T2DM), and impaired glucose tolerance (IGT) among adolescent and adult population.

Methods: PubMed, Cochrane's Library, Science Direct, Scopus, Google Scholar, and ISI Web of Science databases were searched for all available literature until January 2019 for studies assessing the association between omentin and diabetes. The Newcastle-Ottawa Quality Assessment Scale was used to assess the quality of each study.

Results: A total of 28 articles met the inclusion criteria and were included in our systematic review and meta-analysis. There was a significant association between serum omentin and diabetes (WMD-1.68; 95\% CI, -2.17 to $-1.19 ; P<0.001$ ). The result of our sub-group analysis based on participants' health status revealed that omentin was significantly lower in T2DM and IGT subjects but not in T1DM ones compared to healthy controls.

Conclusion: We found that serum omentin level is significantly lower in T2DM and IGT patients but not in T1DM ones. These data could be used by clinicians for early diagnosis and management of diabetes. Furthermore, we need more clinical trials to investigate new agents which could influence omentin levels.
\end{abstract}

Keywords: omentin, diabetes, impaired glucose tolerance, systematic review, meta-analysis

\section{Introduction}

It is expected for diabetes prevalence to increase from 171 million individuals in 2000 to 366 million by $2030 .{ }^{1}$ Diabetes mellitus could be explained best as a complex, multifactorial, chronic metabolic, and endocrine disorder. It has been traditionally defined by the existence of hyperglycemia that can be produced by flaws in insulin secretion, insulin action, or both. ${ }^{2}$ Individuals with diabetes have increased risk of other co-existing complications including myocardial infarction or stroke. ${ }^{3}$ Accordingly, sufficient attention to diabetes is needed to be early diagnosed and treated to decrease its undesirable effects.

Recently, adipose tissue has attracted a lot of attention because of its crucial role in human metabolic pathways. Like an endocrine organ, adipose tissue releases a variety of adipokines including leptin, adiponectin, visfatin, TNF-a, and IL-6 [1,2]. These adipokines could control carbohydrate and lipid metabolism and appear to play a vital role in the pathogenesis of insulin resistance, diabetes, inflammation,
Correspondence: Hossein Hajianfar Food Safety Research Center (SALT) Semnan University of Medical Sciences, Semnan 8I834, Iran

Tel +989131197206

Email hossein.hajain2009@gmail.com 
atherosclerosis, and vascular endothelial dysfunction. ${ }^{4-10}$ Identification of a novel adipokine associated with diabetes might provide new opportunities for clinicians for early diagnosis and better management of diabetes and its related complications. Omentin (intelectin, intestinal lactoferrin receptor, endothelin lectin HL-1, galactofuranosebinding lectin) is a novel fat depot-specific adipokine identified by Yang et al, in 2003 from a visceral omental adipose tissue cDNA library. ${ }^{11}$ There are mainly two isoforms of omentin, omentin-1 and omentin-2, with omentin-1 being the major circulating form in human plasma. ${ }^{12}$ Omentin is an anti-inflammatory adipokine and plays a crucial role in regulating insulin sensitivity through paracrine and endocrine factors where it could enhance insulin sensitivity and glucose metabolism. ${ }^{13}$ Omentin could only hasten insulin-mediated glucose transport and has no modulating effect on the basal glucose transport which designates that it has no inherent insulin-like activity. ${ }^{14}$ Based upon previous reports, plasma omentin levels inversely correlate with body mass index (BMI), fat mass, and fasting plasma insulin, and positively with insulin sensitivity, adiponectin, high-density lipoprotein cholesterol, and endothelial function. ${ }^{15-18}$

However, increasing body of literature has shown paradoxical relationships between serum omentin and diabetes with some studies suggesting a significant association between serum omentin level and diabetes ${ }^{19-23}$ while others do not support such an association. ${ }^{18,24-27}$ Currently, there is insufficient evidence showing whether serum omentin is related to diabetes, and determination of this relationship has rarely been conducted. To address these issues, we carried out this systematic review and meta-analysis by pooling the results from observational studies to examine the association between serum omentin and different types of diabetes including type 1 diabetes mellitus (T1DM), type 2 diabetes mellitus (T2DM), and impaired glucose tolerance (IGT) among adolescent and adult population.

\section{Methods and materials}

This study was performed according to the Preferred Reporting Items for Systematic Reviews and Meta-analysis (PRISMA) statement. ${ }^{28}$

\section{Data source and search strategy}

We searched databases including PubMed, Scopus, Cochrane Library, Science direct, and ISI web of sciences up to January 2019 to identify relevant studies. The reference lists of the included articles were also reviewed to identify additional eligible studies. In order to increase the power of our search strategy and minimize the chance of missing relevant articles, we also contacted expert scientists in the field of diabetes and omentin. The following search strategy was run in PubMed and tailored to each database when necessary: "Diabetes" OR "T2DM" OR "T1DM" OR "glucose tolerance" OR "NIDDM" OR “impaired glucose” OR “impaired fasting glucose" OR "prediabetes" OR “dysglycemia" OR "Diabetes Mellitus" OR "type 2 Diabetes Mellitus" OR "Hyperglycemia" OR "Glucose Tolerance Test" OR "Glucose Intolerance” OR "hyperglycemia” AND “omentin”.

\section{Inclusion criteria}

To be included in the study, publications investigating the association between omentin and diabetes had to meet the following criteria: (1) original articles which examined the association between omentin and glucose metabolism dysfunction including T1DM, T2DM, and IGT; (2) human studies with no restrictions on study parameters (study duration, design, or sample size); (3) adequate data to calculate a relevant measure of association [mean difference, median (Range), and median (interquartile range)]; (4) articles published in English.

\section{Data extraction}

Pairs of independent reviewers screened the titles and abstracts of each study prior to full-text screening of candidate studies. Any discrepancies in terms of decision on a given study were dealt with via discussion and if necessary, arbitration by a third reviewer. For all included studies, two reviewers independently extracted information including first author's name, year of publication, country, sample size, participants' age, gender, BMI, health status, study design, and omentin assay method.

\section{Study quality}

The Newcastle-Ottawa Quality Assessment Scale (NOS) was used to assess the quality of included studies. ${ }^{29}$ The scale consists of the assessment of three domains: selection, comparability, and outcome for a total score of 10 points for cross sectional and 9 points for case-control studies. Studies scoring 7-10, 3-6, and 0-3 points were identified as high, moderate, and low quality, respectively. ${ }^{30}$

\section{Statistical analysis}

Statistical analyses were carried out using the STATA software (version 11.0; Stata Corporation). All data were 
collected as means \pm standard deviation (SD) to estimate the pooled effects. Meta-analysis was performed using hedges'g with 95\% confidence intervals (CIs) for assessing the association between serum omentin levels and diabetes. All analyses were done using the random effects model which takes the between-study variability into account. ${ }^{31}$ The sensitivity analyses were also performed to assess the influence of each individual study on the stability of the meta-analysis results. Each time, one study was excluded to show the impact of that study on the combined effect estimate. We also conducted subgroup analyses based on different factors, including BMI, coexisting disease, participants' health status, and studies location.

\section{Assessment of heterogeneity}

Heterogeneity of the study results was estimated by the chi-squared $\left(\chi^{2}\right)$ test and quantified using the $\mathrm{I}^{2}$ statistic, which represents the percentage of total variation across studies attributable to heterogeneity rather than to chance. $\mathrm{I}^{2}$ was calculated using the formula: $\mathrm{I}^{2}=100 \% \times(\mathrm{Q}-\mathrm{df}) / \mathrm{Q}($ where $\mathrm{Q}$ is the chi-squared statistic, and df is the degree of freedom), and an $\mathrm{I}^{2}$ value of $75 \%$ or greater was deemed to indicate a high level of inconsistency. Significant heterogeneity was defined as a $P$-value of $<0.05$. $^{30}$

\section{Assessment of publication bias}

Publication bias was assessed by visual inspection of the funnel plots, and Egger's and Begg's tests were conducted to determine the degree of funnel plot asymmetry with $p<0.05$ representing significant publication bias. ${ }^{30}$ When publication bias was found, trim \& fill analysis performed to find out the effects of missed study on overall effects.

\section{Results}

\section{Search results}

Our initial search through databases identified a total of 4675 articles. Removing duplicates yielded 3857 articles which were reviewed based on the title and abstract by two independent reviewers and 3039 irrelevant studies were excluded at this stage. Ninety-seven papers were retrieved and reviewed based on full text, and 28 articles met the inclusion criteria and were included in our systematic review and meta-analysis. The PRISMA flow diagram summarizes the results of the study selection process for this systematic review and meta-analysis (Figure 1).

\section{Overview of included studies}

The general characteristics of the included studies are shown in Table 1. A total of 28 studies involving 3354 participants were included in our systematic review and meta-analysis with their sample size ranging from 37 to 496 subjects. Selected studies were published between 2008 and 2018. Mean age of the participants ranged between 11 and 67 years. Twenty-one studies recruited both gender, ${ }^{17,19,22-24,27,32-46}$ four were conducted only in men, ${ }^{18,21,25,47}$ and three used female subjects. ${ }^{20,26,48}$ Included studies were conducted in participants with $\mathrm{T} 1 \mathrm{DM},{ }^{17,32,40} \mathrm{~T} 2 \mathrm{DM}^{22,23,26,27,38,39,41,43-47}$, and patients with IGT. $^{18,21,24,42}$ Seven studies enrolled normal weight $(\mathrm{BMI}<24.9)$ subjects, ${ }^{23,32,33,36,44-46}$ 13 recruited overweight participants $(24.9 \leq \mathrm{BMI} \leq 29.9)^{17,18,21,22,25,27,34,38,39,41,43,47,48}$ and 6 were conducted on obese subjects ${ }^{20,24,26,35,37,42}$ but 2 studies did not mention baseline BMI. ${ }^{19,40}$ Two studies were cross sectional ${ }^{35,37}$ and the others were casecontrol. ${ }^{17-27,32-34,36,38-48}$ Based on the NOS, 10 studies ranked as high-quality ${ }^{23,27,32,35,37,40,41,43,44,47}$ and the others as were classified as moderate in quality assessment.

\section{The association between serum omentin level and diabetes}

Twenty-eight studies with 2941 participants examined serum omentin level among patients with diabetes (T1DM, T2DM, and IGT) and healthy subjects. ${ }^{17-27,32-48}$ There was a significant association between serum omentin and diabetes (WMD-1.68; 95\% CI, -2.17 to -1.19 ; $P<0.001)$. There was evidence of heterogeneity between the effect sizes of the included studies $\left(\mathrm{I}^{2}=96.9 \%\right.$ $p<0.001$ ). So, we did sub-group analysis based on the BMI category (normal/overweight/obese), participants' health status (T1DM/T2DM/IGT), co-existing disease (diabetes/diabetes plus other complications), and location (Asia/Europe/Africa). None of these sub-group analyses could reduce heterogeneity. The result of our sub-group analysis based on participants' health status revealed that omentin was significantly lower in T2DM and IGT subjects but not in T1DM ones compared to healthy controls. The results of the sub-group analysis are shown in Table 2. There was evidence of publication bias (Begg's test: $P<0.001$, Egger's test: $P<0.001$ ), therefore, we did trim $\&$ fill analysis to find out probable missed studies but this method could not add any studies to our included ones 


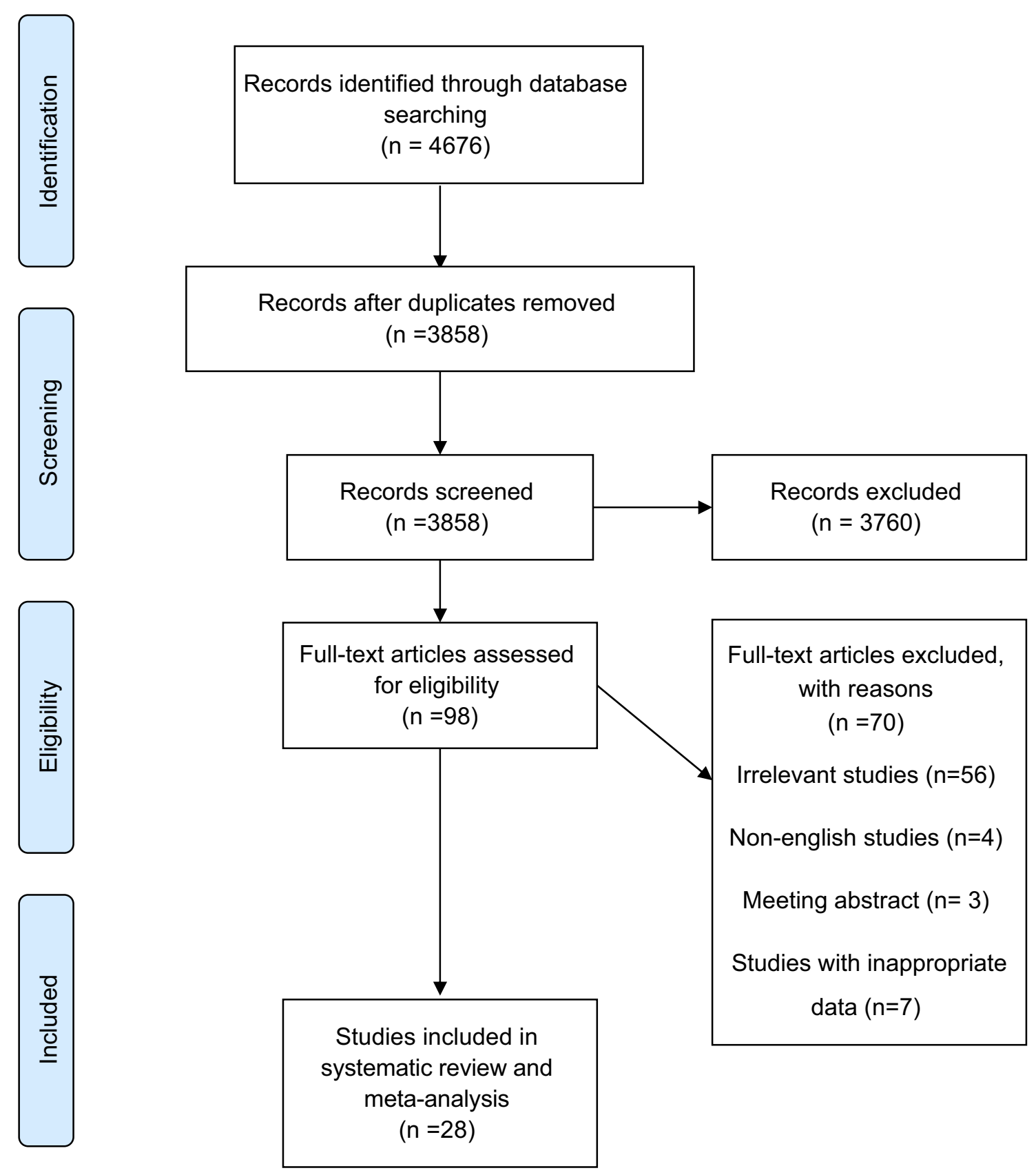

Figure I The flow diagram of study selection.

(Figure 2). The sensitivity analysis showed that removing any of the studies could not substantially change the association between omentin and diabetes.

\section{Discussion}

In order to identify new biomarkers for early diagnosis of diabetes and better management of its progress and complications, various studies have been conducted. However, interpreting the literature to wrap up a final conclusion is such a difficult process for clinicians, comprehensive systematic review and meta-analysis of available literature can represent the most reliable evidence. ${ }^{49}$

The present study included 28 observational studies which involved a total of 3354 individuals. Present study showed that serum level of omentin was significantly lower in T2DM and IGT subjects but not in T1DM ones compared to healthy controls. Furthermore, sub-group analysis could not reduce heterogeneity or change the overall results. Although overall results revealed a significant association between omentin with T2DM and IGT, there 
Table I Characteristics of included studies

\begin{tabular}{|c|c|c|c|c|c|c|c|c|}
\hline Author, year & Location & $\begin{array}{l}\text { Sample } \\
\text { size }(F / M)\end{array}$ & Age range & BMI & Study design & $\begin{array}{l}\text { Assay } \\
\text { method }\end{array}$ & $\begin{array}{l}\text { Health } \\
\text { status }\end{array}$ & $\begin{array}{l}\text { Quality } \\
\text { score }\end{array}$ \\
\hline $\begin{array}{l}\text { Abd-Elbaky et al., } \\
2016^{47}\end{array}$ & Egypt & $160 M$ & $40.3 \pm 3.6$ & $26.7 \pm 0.55$ & Case-control & ELISA & T2DM & $7 / 9$ \\
\hline Zhang et al., $2014^{27}$ & China & $58 / 62$ & $66.53 \pm 10.68$ & $26.07 \pm 2.20$ & Case-control & ELISA & T2DM & $7 / 9$ \\
\hline Yoo et al., $2011^{46}$ & Korea & $53 / 37$ & $54.54 \pm 6.99$ & $24.02 \pm 2.42$ & Case-control & ELISA & T2DM & $6 / 9$ \\
\hline Yan et al., $2011^{23}$ & China & $51 / 54$ & $53.8 \pm 1.6$ & $24.07 \pm 0.49$ & Case-control & ELISA & T2DM & $6 / 9$ \\
\hline Yan et al., $2011^{45}$ & China & $35 / 25$ & $51.6 \pm 8.45$ & $24.05 \pm 3.75$ & Case-control & ELISA & T2DM & $7 / 9$ \\
\hline Wan et al., $2015^{44}$ & China & $68 / 57$ & $58.26 \pm 7.95$ & $23.8 \pm 2.29$ & Case-control & ELISA & $\begin{array}{l}\text { T2DM + } \\
\text { Retinopathy }\end{array}$ & $7 / 9$ \\
\hline $\begin{array}{l}\text { Urbanova et al., } \\
2014^{26}\end{array}$ & Czech & $37 \mathrm{~F}$ & $49.85 \pm 2.5$ & $37.55 \pm 1.47$ & Case-control & ELISA & T2DM & $4 / 9$ \\
\hline Tekce et al., $2014^{43}$ & Turkey & $39 / 52$ & $54.9 \pm 10.8$ & $25.7 \pm 3.55$ & Case-control & ELISA & T2DM + CKD & $8 / 9$ \\
\hline Tan et al., $2008^{17}$ & UK & $39 \mathrm{M} \& \mathrm{~F}$ & $30.2 \pm 4.5$ & $25.5 \pm 3.1$ & Case-control & ELISA & TIDM & $4 / 9$ \\
\hline Sperling et al., $2016^{42}$ & Poland & $33 / 31$ & $30-60$ & $38.95 \pm 6.05$ & Case-control & ELISA & IGT & $4 / 9$ \\
\hline Pan et al., $2010^{41}$ & China & $68 / 83$ & $37.99 \pm 6.02$ & $25.7 \pm 3.74$ & Case-control & ELISA & T2DM & $7 / 9$ \\
\hline Nurten et al., $2018^{40}$ & Germany & $213 / 283$ & $11.35 \pm 4.17$ & - & Case-control & ELISA & TIDM & $7 / 9$ \\
\hline Motawi et al., $2018^{22}$ & Egypt & $72 / 48$ & $54.95 \pm 4.55$ & $27.15 \pm 1.55$ & Case-control & ELISA & T2DM + CAS & $6 / 9$ \\
\hline $\begin{array}{l}\text { Moreno-Navarrete } \\
\text { et al., } 201 \mathrm{I}^{18}\end{array}$ & Spain & $248 M$ & $53.35 \pm 10.85$ & $27.75 \pm 3.5$ & Case-control & ELISA & IGT & $6 / 9$ \\
\hline Matloch et al., $2018^{39}$ & Czech & $8 / 30$ & $67.45 \pm 8.76$ & $29.05 \pm 4.34$ & Case-control & ELISA & T2DM + CAD & $4 / 9$ \\
\hline $\begin{array}{l}\text { Kocijancic et al., } \\
2015^{38}\end{array}$ & Croatia & $84 / 36$ & $64.37 \pm 13.62$ & $25.25 \pm 4$ & Case-control & ELISA & T2DM + HD & $5 / 9$ \\
\hline Gursoy et al., $2010^{48}$ & Turkey & $120 \mathrm{~F}$ & $53.75 \pm 9.45$ & $29.3 \pm 5.25$ & Case-control & ELISA & T2DM & $6 / 9$ \\
\hline Kahwaji et al., $2017^{37}$ & Jordan & $13 \mid / 64$ & $51.26 \pm 10.45$ & $33.32 \pm 5.47$ & Cross sectional & - & T2DM + MetS & $7 / 10$ \\
\hline Nassif et al., $2013^{36}$ & Egypt & $15 / 65$ & $43.15 \pm 1.77$ & $23.9 \pm 0.37$ & Case-control & ELISA & $\mathrm{T} 2 \mathrm{DM}+\mathrm{HCV}$ & $4 / 9$ \\
\hline Greulich et al., $2013^{25}$ & Germany & $92 \mathrm{M}$ & $55.5 \pm 6.35$ & $27.85 \pm 3$ & Case-control & ELISA & T2DM & $5 / 9$ \\
\hline Gateva et al., $2018^{24}$ & Bulgaria & $68 / 12$ & $50.4 \pm 10.6$ & $37.15 \pm 5.65$ & Case-control & ELISA & IGT & $4 / 9$ \\
\hline Flehmig et al., $2014^{35}$ & Germany & $74 / 67$ & $48 \pm 14$ & $46.1 \pm 10.1$ & Cross sectional & ELISA & T2DM & $7 / 10$ \\
\hline Elsaid et al., $2018^{20}$ & Egypt & $90 \mathrm{~F}$ & $46.6 \pm 6.7$ & $30.45 \pm 4.55$ & Case-control & ELISA & T2DM & $5 / 9$ \\
\hline Dogan et al., $2016^{33}$ & Turkey & $32 / 28$ & $48.5 I \pm 5.45$ & $22.69 \pm 1.54$ & Case-control & ELISA & $\mathrm{T} 2 \mathrm{DM}+\mathrm{CP}$ & $5 / 9$ \\
\hline Ahmed et al., $2018^{19}$ & Egypt & $100 \mathrm{M} \& \mathrm{~F}$ & - & - & Case-control & ELISA & T2DM + CVD & $4 / 9$ \\
\hline $\begin{array}{l}\text { Abd El Dayem et al., } \\
2015^{32}\end{array}$ & Egypt & $46 / 46$ & $16.22 \pm 2.07$ & $24.83 \pm 4.93$ & Case-control & ELISA & TIDM & $7 / 9$ \\
\hline $\begin{array}{l}\text { El-Mesallamy et. al., } \\
20 \mathrm{II}^{34}\end{array}$ & Egypt & $23 / 67$ & $56.53 \pm 2$ & $28.33 \pm 0.53$ & Case-control & ELISA & T2DM + IHD & $6 / 9$ \\
\hline Kaushik et al., $2018^{21}$ & India & $150 \mathrm{M}$ & $4 I . I \pm 8.03$ & $26.7 I \pm 3.09$ & Case-control & ELISA & IGT & $5 / 9$ \\
\hline
\end{tabular}

Abbreviations: BMI, body mass index; ELISA, enzyme-linked immunosorbent assay; T2DM, type 2 diabetes mellitus; TIM, type I diabetes mellitus; IGT, impaired glucose tolerance; CKD, chronic kidney disease; CAS, coronary artery stenosis; CAD, coronary artery disease; HD, hemodialysis; MetS, metabolic syndrome; CAN, cardiac autonomic neuropathy; HCV, hepatitis C virus; CP, chronic periodontitis; LAD, latent autoimmune diabetes; CVD, cardiovascular disease; IHD, ischemic heart disease.

are inconsistencies among included studies which should be taken into account when interpreting the results. We found significant heterogeneity in our study, which might be contributed to different population, sample size, omentin assay kit, participants' BMI, and age, presence of other co-existing diseases, subjects' health status and location of studies.

Our analysis failed to show any significant association between omentin and T1DM. The results of previous studies in this sub-group are inconsistent. In other words, some evidence proved lower level of omentin in T1DM subjects $^{17,22,32}$ but Nurten et al, revealed higher levels of this adipokine in these populations. ${ }^{40}$ The included studies of this sub-group had high ${ }^{32,40}$ and moderate quality. ${ }^{17,22}$ Another sub-group of our study included T2DM patients. The analysis proved lower levels of omentin in these populations which is consistent with previous reports, ${ }^{19,20,33,45}$ but was not confirmed by some other evidence. ${ }^{35,39}$ The quality of included studies was high $23,27,35,37,41,43,44,47,50-52$ or moderate. ${ }^{12,19,20,25,26,33,34,36,38,39,45,46,48,53,54}$ The last 
Table 2 Result of sub-group analysis of included studies in meta-analysis

\begin{tabular}{|c|c|c|c|c|c|c|}
\hline Sub-grouped by & $\begin{array}{l}\text { No. of } \\
\text { trials }\end{array}$ & $\begin{array}{l}\text { Effect } \\
\text { size }^{a}\end{array}$ & $95 \% \mathrm{Cl}$ & $\begin{array}{l}I^{2} \\
(\%)\end{array}$ & $\begin{array}{l}P \text { for } \\
\text { heterogeneity }\end{array}$ & $\begin{array}{l}P \text { for between sub-group } \\
\text { heterogeneity }\end{array}$ \\
\hline \multicolumn{7}{|l|}{ BMI category } \\
\hline Normal weight $\left(\leq 24.9 \mathrm{~kg} / \mathrm{m}^{2}\right)$ & 12 & -1.63 & $-2.45,-0.81$ & 96.9 & $<0.001$ & \multirow[t]{3}{*}{$<0.001$} \\
\hline $\begin{array}{l}\text { Overweight } \\
(24.9<\text { BMI } \leq 29.9)\end{array}$ & 13 & -2.09 & $-2.92,-1.26$ & 97.1 & $<0.001$ & \\
\hline Obese (BMI $\geq 30$ ) & 6 & -0.91 & $-1.8,-0.03$ & 95.5 & $<0.001$ & \\
\hline \multicolumn{7}{|l|}{ Health status } \\
\hline TIDM & 5 & -1.35 & $-2.94,0.23$ & 98.3 & $<0.001$ & \multirow[t]{3}{*}{$<0.001$} \\
\hline T2DM & 22 & -1.98 & $-2.59,-1.36$ & 96.4 & $<0.001$ & \\
\hline IGT & 4 & -0.60 & $-1.11,-0.08$ & 83.4 & $<0.001$ & \\
\hline \multicolumn{7}{|l|}{ Co-existing disease } \\
\hline Diabetes & 23 & -1.44 & $-1.99,-0.88$ & 97 & $<0.001$ & \multirow[t]{2}{*}{$<0.001$} \\
\hline $\begin{array}{l}\text { Diabetes plus other } \\
\text { complications }\end{array}$ & 8 & -2.51 & $-3.68,-1.34$ & 96.5 & $<0.001$ & \\
\hline \multicolumn{7}{|l|}{ Location } \\
\hline Asia & 8 & -1.19 & $-1.75,-0.63$ & 92.2 & $<0.001$ & \multirow[t]{3}{*}{$<0.001$} \\
\hline Europe & 15 & -0.92 & $-1.45,-0.38$ & 95.2 & $<0.001$ & \\
\hline Africa & 8 & -3.32 & $-4.49,-2.15$ & 95.2 & $<0.001$ & \\
\hline
\end{tabular}

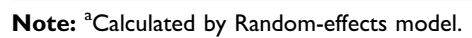

Abbreviations: BMI, body mass index; TIDM, type I diabetes mellitus; T2DM, type 2 diabetes mellitus; IGT, impaired glucose tolerance.

sub-group consisted of patients diagnosed with IGT. Our result revealed that omentin level was significantly lower in these subjects and was consistent with previous evidence, ${ }^{21}$ although was not approved by other ones. ${ }^{18,24}$ It should be stated that all the included studies in this sub-group ranked as moderate quality. ${ }^{18,21,24,42}$ The possible explanation for discrepancies among available literature in each sub-group may be contributed to small sample size, different ethnicity, gender, and stages of diabetes. Insignificant association between omentin and T1DM could be attributed to primary absolute insulin deficiency seen in T1DM patients compared with T2DM patients which is due to lower omentin-1 levels ${ }^{55}$ and higher BMI levels in T2DM subjects compared to T1DM patients. ${ }^{56}$

In recent years, visceral adipose tissue and adipokines have attracted lots of attention. Omentin, a novel adipokine, is mainly expressed in visceral adipose tissue and has been suggested as a biomarker of metabolic disease. ${ }^{57}$ Based on previous evidence, serum omentin levels were negatively associated with metabolic risk factors. Also, it has been proved that omentin may play a role as an antiinflammatory and insulin-sensitizing agent. ${ }^{18,58}$ Recently, it has been documented that omentin may be associated with vascular function modulation by endothelium-dependent vasodilation. Recent mechanism may explain the cardioprotective effects of omentin. ${ }^{18,59}$ Omentin exerts its anti-atherosclerotic properties by supersession of cytokine-stimulated expression of adhesion molecules in endothelial cells. $^{60}$ In vitro studies illustrated that Omentin as a polypeptide hormone increases insulin-stimulated glucose uptake and also Akt phosphorylation in human adipocytes. ${ }^{14}$ The Akt pathway is a signal transduction pathway which enhances survival and growth in reaction to extracellular signals. The dysfunction of Akt pathway regulation results in increased signaling activity which could lead to cancer and T2DM. ${ }^{61}$ By activating protein kinase $\mathrm{B}$, omentin boosts insulin signal transduction and enhances insulin-mediated glucose transport in adipocytes. ${ }^{14}$ Decreased serum omentin level, observed in T2DM patients, could cause a reduction in insulinstimulated glucose uptake in insulin-sensitive tissues. This may explain the state of insulin resistance present in T2DM. ${ }^{27,41}$ It has been reported that glucose and insulin could decrease mRNA expression of omentin and omentin protein production in adipose tissue. This mechanism may indicate that plasma glucose and insulin levels directly or 


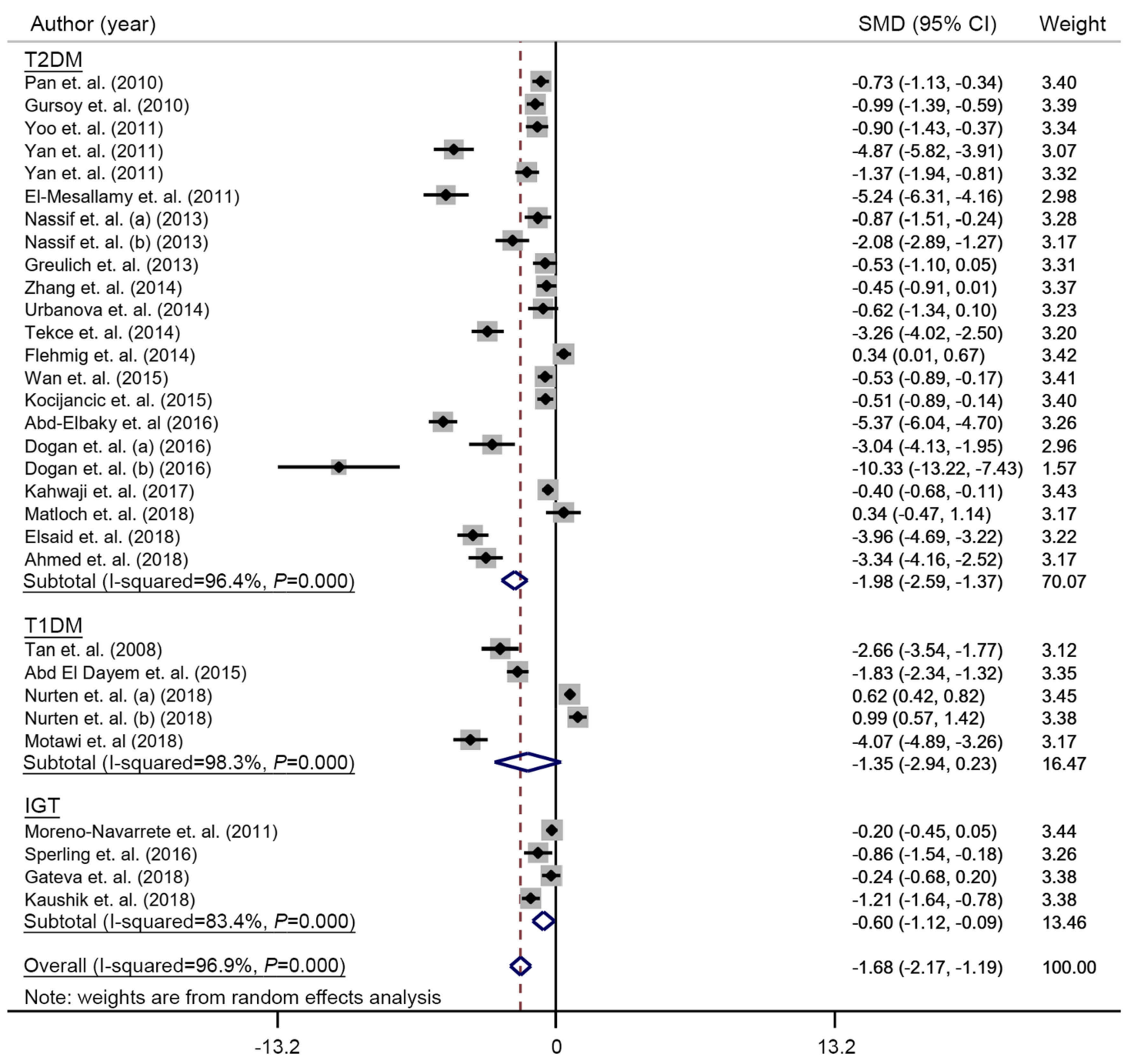

Figure 2 Forest plot of the association between the serum omentin levels and diabetes sub-grouped by types of diabetes. Abbreviations: T2DM, type 2 diabetes mellitus; TIDM, type I diabetes mellitus; IGT, impaired glucose tolerance.

indirectly modulate omentin synthesis. ${ }^{17}$ On the other hand, serum omentin may influence the glucose or insulin levels. So we speculate that the association between omentin, insulin, and glucose might be a mutual relationship. ${ }^{27}$

The present study has some limitations that warrant consideration. First, significant heterogeneity was present in our analysis that would limit the generalization of our findings. Heterogeneity between studies may be explained by different population, sample size, omentin assay kit, participants' BMI and age, presence of other co-existing disease, subjects' health status and location of studies. Moreover, five of the included studies used a cross-sectional design..$^{35,37,50-52}$ The nature of cross-sectional studies makes it impossible to draw a causal link between variables. Since, it is a snapshot of the population, it could be altered overtime and included
Neyman's bias (prevalence-incidence bias), which is another form of selection bias and highlighted in longer-lasting disorders. ${ }^{62}$ Other studies used case-control design which is prone to selection bias. ${ }^{63}$

There is no previous systematic review and meta-analysis assessing the link between serum omentin level and diabetes including T1DM, T2DM, and IGT in observational studies which could be considered as our study strength.

\section{Conclusion}

According to what was discussed, we found that serum omentin level is significantly lower in T2DM and IGT patients but not in T1DM ones. These data could be used by clinicians for early diagnosis and management of 
diabetes. Furthermore, we need more clinical trials to investigate new agents with possible influence on omentin levels.

\section{Author contributions}

All authors contributed to data analysis, drafting or revising the article, gave final approval of the version to be published, and agree to be accountable for all aspects of the work.

\section{Disclosure}

The authors declare no conflicts of interest in this work.

\section{References}

1. Wild S, Roglic G, Green A, Sicree R, King H. Global prevalence of diabetes: estimates for the year 2000 and projections for 2030. Diabetes Care. 2004;27(5):1047-1053. doi:10.2337/diacare.27.5.1047

2. Association AD. Standards of medical care in diabetes-2014. Diabetes Care. 2014;37(Supplement 1):S14-S80.

3. Almdal T, Scharling H, Jensen JS, Vestergaard H. The independent effect of type 2 diabetes mellitus on ischemic heart disease, stroke, and death: a population-based study of 13000 men and women with 20 years of follow-up. Arch Intern Med. 2004;164(13):1422-1426. doi:10.1001/archinte.164.13.1422

4. Wannamethee SG, Lowe GD, Rumley A, Lynne CL, Whincup PH, Sattar N. Adipokines and risk of type 2 diabetes in older men. Diabetes Care. 2007. doi: $10.2337 / \mathrm{dc} 06-2416$

5. Ahima RS, Lazar MA. Adipokines and the peripheral and neural control of energy balance. Mol Endocrinol. 2008;22(5):1023-1031. doi:10.1210/me.2007-0424

6. Hivert M-F, Sullivan LM, Fox CS, et al. Associations of adiponectin, resistin, and tumor necrosis factor- $\alpha$ with insulin resistance. $J$ Clin Endocrinol Metab. 2008;93(8):3165-3172. doi:10.1210/jc.2008-0425

7. Lorenzo M, Fernández-Veledo S, Vila-Bedmar R, Garcia-Guerra L, De Alvaro C, Nieto-Vazquez I. Insulin resistance induced by tumor necrosis factor- $\alpha$ in myocytes and brown adipocytes. J Anim Sci. 2008;86(supp1_14):E94-E104.

8. Rehman K, Akash MSH. Mechanisms of inflammatory responses and development of insulin resistance: how are they interlinked? $J$ Biomed Sci. 2016;23(1):87. doi:10.1186/s12929-016-0303-y

9. Rehman K, Akash MSH, Liaqat A, Kamal S, Qadir MI, Rasul A. Role of interleukin-6 in development of insulin resistance and type 2 diabetes mellitus. Crit Rev TM Eukaryotic Gene Expression. 2017;27:3.

10. Akash MSH, Rehman K, Liaqat A. Tumor necrosis factor-alpha: role in development of insulin resistance and pathogenesis of type 2 diabetes mellitus. $J$ Cell Biochem. 2018;119(1):105-110. doi:10.1002/jcb.27261

11. Yang R, Xu A, Pray J, et al. Cloning of omentin, a new adipocytokine from omental fat tissue in humans. Diabetes. 2003;52:A1.

12. Chen WJY, Greulich S, Rijzewijk LJ, et al. Plasma omentin levels in relation to cardiac function in patients with type 2 diabetes and healthy controls: effect of pioglitazone versus metformin. Diabetologia. 2011;54:S101-S101. doi:10.1007/s00125-010-2040-1

13. Senthilkumar GP, Anithalekshmi MS, Yasir M, Parameswaran S, Muthu Packirisamy R, Bobby Z. Role of omentin 1 and IL-6 in type 2 diabetes mellitus patients with diabetic nephropathy. Diabetes Metab Syndrome. 2018;12(1):23-26. doi:10.1016/j.dsx.2017.08.005

14. Yang R-Z, Lee M-J, Hu H, et al. Identification of omentin as a novel depot-specific adipokine in human adipose tissue: possible role in modulating insulin action. Am J Physiol Endocrinol Metab. 2006;290 (6):E1253-E1261. doi:10.1152/ajpendo.00484.2005
15. de Souza Batista CM, Yang R-Z, Lee M-J, et al. Omentin plasma levels and gene expression are decreased in obesity. Diabetes. 2007;56(6):1655-1661. doi:10.2337/db06-1506

16. Pan H-Y, Guo L, Li Q. Changes of serum omentin-1 levels in normal subjects and in patients with impaired glucose regulation and with newly diagnosed and untreated type 2 diabetes. Diabetes Res Clin Pract. 2010;88(1):29-33. doi:10.1016/j.diabres.2010.01.013

17. Tan BK, Pua S, Syed F, Lewandowski KC, O’Hare JP, Randeva HS. Decreased plasma omentin-1 levels in type 1 diabetes mellitus. Diabet Med. 2008;25(10):1254-1255. doi:10.1111/dme.2008.25.issue-10

18. Moreno-Navarrete JM, Ortega F, Castro A, Sabater M, Ricart W, Fernandez-Real JM. Circulating omentin as a novel biomarker of endothelial dysfunction. Obesity. 2011;19(8):1552-1559. doi:10.1038/ oby. 2010.204

19. Ahmed HH, Shousha WG, El-mezayen HA, Emara IA, Hassan ME. New biomarkers as prognostic factors for cardiovascular complications in type 2 diabetic patients. Indian J Clinical Biochem. 2018;1-9.

20. Elsaid NH, Sadik NA, Ahmed NR, Fayez SE, Mohammed NA. Serum omentin-1 levels in type 2 diabetic obese women in relation to glycemic control, insulin resistance and metabolic parameters. J Clin Transl Endocrinol. 2018;13:14-19. doi:10.1016/j.jcte.2018.05.003

21. Kaushik N, Kaushik R, Dixit P, et al. Plasma omentin-1 level and its relationship with insulin resistance in obese prediabetics. $J$ Clin Diagn Res. 2018;12(4):BC10-BC14.

22. Motawi TMK, Mahdy SG, El-Sawalhi MM, Ali EN, El-Telbany RFA. Serum levels of chemerin, apelin, vaspin, and omentin-1 in obese type 2 diabetic Egyptian patients with coronary artery stenosis. Can J Physiol Pharmacol. 2018;96(1):38-44. doi:10.1139/cjpp-2017-0272

23. Yan $\mathrm{P}$, Li L, Yang M, et al. Effects of the long-acting human glucagon-like peptide-1 analog liraglutide on plasma omentin-1 levels in patients with type 2 diabetes mellitus. Diabetes Res Clin Pract. 2011;92(3):368-374. doi:10.1016/j.diabres.2011.02.030

24. Gateva A, Assyov Y, Tsakova A, Kamenov Z. Classical (adiponectin, leptin, resistin) and new (chemerin, vaspin, omentin) adipocytokines in patients with prediabetes. Horm Mol Biol Clin Investig. 2018;34:1.

25. Greulich S, Chen WJ, Maxhera B, et al. Cardioprotective properties of omentin-1 in type 2 diabetes: evidence from clinical and in vitro studies. PLoS One. 2013;8(3):e59697. doi:10.1371/journal. pone. 0059697

26. Urbanová M, Dostálová I, Trachta $\mathrm{P}$, et al. Serum concentrations and subcutaneous adipose tissue mRNA expression of omentin in morbid obesity and type 2 diabetes mellitus: the effect of very-low-calorie diet, physical activity and laparoscopic sleeve gastrectomy. Physiol Res. 2014;63:2.

27. Zhang Q, Zhu L, Zheng M, et al. Changes of serum omentin-1 levels in normal subjects, type 2 diabetes and type 2 diabetes with overweight and obesity in Chinese adults. Annales D'endocrinologie. 2014;75(3):171-175. doi:10.1016/j.ando.2014.04.013

28. Moher D, Shamseer L, Clarke M, et al. Preferred reporting items for systematic review and meta-analysis protocols (PRISMA-P) 2015 statement. Syst Rev. 2015;4(1):1. doi:10.1186/2046-4053-4-1

29. Wells G. The Newcastle-Ottawa Scale (NOS) for assessing the quality of nonrandomised studies in meta-analysis. Available from: http://www.ohri. ca/programs/clinical_epidemiology/oxford.asp. Accessed July 3, 2019.

30. Arab A, Rafie N, Mansourian M, Miraghajani M, Hajianfar H. Dietary patterns and semen quality: a systematic review and metaanalysis of observational studies. Andrology. 2018;6(1):20-28. doi:10.1111/andr.12430

31. Arab A, Rafie N, Mansourian M, Miraghajani M, Hajianfar H. Dietary patterns and semen quality: a systematic review and meta-analysis of observational studies. Andrology. 2018;6(1):20-28. doi:10.1111/ andr. 12430

32. Abd El Dayem SM, Battah AA, El Shehaby A. Cardiac affection in type 1 diabetic patients in relation to omentin. Open Access Macedonian J Med Sci. 2015;3(4):699-704. doi:10.3889/ oamjms.2015.132 
33. Dogan SB, Dede FO, Balli U, Sertoglu E. Levels of vaspin and omentin-1 in gingival crevicular fluid as potential markers of inflammation in patients with chronic periodontitis and type 2 diabetes mellitus. J Oral Sci. 2016;58 (3):379-389. doi:10.2334/josnusd.15-0731

34. El-Mesallamy HO, El-Derany MO, Hamdy NM. Serum omentin-1 and chemerin levels are interrelated in patients with type 2 diabetes mellitus with or without ischaemic heart disease. Diabet Med. 2011;28(10):1194-1200. doi:10.1111/j.1464-5491.2011.03281.x

35. Flehmig G, Scholz M, Kloting N, et al. Identification of adipokine clusters related to parameters of fat mass, insulin sensitivity and inflammation. PLoS One. 2014;9(6):e99785. doi:10.1371/journal.pone.0099785

36. Hussein Nassif WM, Ismail Amin A, Abdeltawab Hassan Z, Abdelhafiz Abdelaziz DH. Changes of serum omentin-1 levels and relationship between omentin-1 and insulin resistance in chronic hepatitis C patients. EXCLI J. 2013;12:924-932.

37. Kahwaji R, Kasabri V, Bulatova N, et al. Evaluation of correlations of plasma levels of oxytocin, omentin-1 and irisin in diabetic and nondiabetic metabolic syndrome patients: a cross sectional study in Jordan. Jordan Med J. 2017;51(3):97-108.

38. Kocijancic M, Vujicic B, Racki S, Cubranic Z, Zaputovic L, Dvornik S. Serum omentin-1 levels as a possible risk factor of mortality in patients with diabetes on haemodialysis. Diabetes Res Clin Pract. 2015;110(1):44-50. doi:10.1016/j.diabres.2015.06.008

39. Matloch Z, Kratochvilova H, Cinkajzlova A, et al. Changes in omentin levels and its mRNA expression in epicardial adipose tissue in patients undergoing elective cardiac surgery: the influence of type 2 diabetes and coronary heart disease. Physiol Res. 2018;67(6):881-890.

40. Nurten E, Vogel M, Kapellen TM, et al. Omentin-1 and NAMPT serum concentrations are higher and CK-18 levels are lower in children and adolescents with type 1 diabetes when compared to healthy age, sex and BMI matched controls. J Pediatr Endocrinol Metab. 2018. doi:10.1515/jpem-2018-0353

41. Pan HY, Guo L, Li Q. Changes of serum omentin-1 levels in normal subjects and in patients with impaired glucose regulation and with newly diagnosed and untreated type 2 diabetes. Diabetes Res Clin Pract. 2010;88(1):29-33. doi:10.1016/j.diabres.2010.01.013

42. Sperling M, Grzelak T, Pelczyńska M, et al. Concentrations of omentin and vaspin versus insulin resistance in obese individuals. Biomed Pharmacother. 2016;83:542-547. doi:10.1016/j.biopha.2016.07.012

43. Tekce H, Tekce BK, Aktas G, Alcelik A, Sengul E. Serum omentin-1 levels in diabetic and nondiabetic patients with chronic kidney disease. Exp Clin Endocrinol Diabetes. 2014;122(8):451-456. doi:10.1055/s-0034-1375674

44. Wan W, Li Q, Zhang F, et al. Serum and vitreous concentrations of omentin-1 in diabetic retinopathy. Dis Markers. 2015;2015:754312. doi: $10.1155 / 2015 / 105358$

45. Yan P, Liu D, Long M, Ren Y, Pang J, Li R. Changes of serum omentin levels and relationship between omentin and adiponectin concentrations in type 2 diabetes mellitus. Exp Clin Endocrinol Diabetes. 2011;119(4):257-263.

46. Yoo HJ, Hwang SY, Hong HC, et al. Association of circulating omentin-1 level with arterial stiffness and carotid plaque in type 2 diabetes. Cardiovasc Diabetol. 2011;10:103.

47. Abd-Elbaky AE, Abo-ElMatty DM, Mesbah NM, Ibrahim SM. Omentin and apelin concentrations in relation to obesity, diabetes mellitus type two, and cardiovascular diseases in Egyptian population. Int $J$ Diabetes Dev Ctries. 2016;36(1):52-58. doi:10.1007/ s13410-015-0416-y
48. Gürsoy G, Kirnap N, Eşbah O, et al. The relationship between plasma omentin-1 levels and insulin resistance in newly diagnosed type 2 diabetıc women. Clin Rev Opinions. 2010;2(4):49-54.

49. Hadi A, Pourmasoumi M, Mohammadi H, Symonds M, Miraghajani M. The effects of silymarin supplementation on metabolic status and oxidative stress in patients with type 2 diabetes mellitus: a systematic review and meta-analysis of clinical trials. Complement Ther Med. 2018;41:311-319.

50. Delitala AP, Sanciu FA, Errigo A, Delitala G, Pes GM. Leptin levels and insulin dependence in latent autoimmune diabetes in adults. $J$ Interferon Cytokine Res. 2017;37(12):550-556.

51. Herder C, Kannenberg JM, Niersmann C, et al. Independent and opposite associations of serum levels of omentin- 1 and adiponectin with increases of glycaemia and incident type 2 diabetes in an older population: KORA F4/FF4 study. Eur J Endocrinol. 2017;177 (4):277-286. doi:10.1530/EJE-17-0100

52. Jung CH, Jung $\mathrm{SH}$, Kim BY, Kim CH, Kang SK, Mok JO. Association of serum omentin levels with cardiac autonomic neuropathy in patients with type 2 diabetes mellitus: a hospital-based study. Cardiovasc Diabetol. 2015;14:140. doi:10.1186/s12933-0150303-3

53. Senthilkumar GP, Anithalekshmi MS, Yasir M, Parameswaran S, Packirisamy RM, Bobby Z. Role of omentin 1 and IL-6 in type 2 diabetes mellitus patients with diabetic nephropathy. Diabetes Metab Syndr. 2018;12(1):23-26. doi:10.1016/j.dsx.2017.08.005

54. Zorlu M, Kiskac M, Guler E, et al. Serum obestatin and omentin levels in patients with diabetic nephropathy. Niger J Clin Pract. 2017;20(2):182-187. doi:10.4103/1119-3077.181350

55. Yan P, Liu D, Long M, Ren Y, Pang J, Li R. Changes of serum omentin levels and relationship between omentin and adiponectin concentrations in type 2 diabetes mellitus. Exp Clin Endocrinol Diabetes. 2011;119(04):257-263. doi:10.1055/s-0030-1269912

56. Bays HE, Chapman R, Grandy S, Group SI. The relationship of body mass index to diabetes mellitus, hypertension and dyslipidaemia: comparison of data from two national surveys. Int J Clin Pract. 2007;61(5):737-747. doi:10.1111/j.1742-1241.2007.01336.x

57. Shibata R, Ouchi N, Takahashi R, et al. Omentin as a novel biomarker of metabolic risk factors. Diabetol Metab Syndr. 2012;4(1):37.

58. Zhou J-Y, Chan L, Zhou S-W. Omentin: linking metabolic syndrome and cardiovascular disease. Curr Vasc Pharmacol. 2014;12(1):136143.

59. Narumi T, Watanabe $\mathrm{T}$, Kadowaki S, et al. Impact of serum omentin-1 levels on cardiac prognosis in patients with heart failure. Cardiovasc Diabetol. 2014;13(1):84. doi:10.1186/1475-284013-84

60. Tan BK, Adya R, Randeva HS. Omentin: a novel link between inflammation, diabesity, and cardiovascular disease. Trends Cardiovasc Med. 2010;20(5):143-148.

61. Carracedo A, Pandolfi PP. The PTEN-PI3K pathway: of feedbacks and cross-talks. Oncogene. 2008;27(41):5527-5541. doi:10.1038/ onc. 2008.247

62. Levin KA. Study design III: cross-sectional studies. Evid-based Dent. 2006;7(1):24-25.

63. Schulz KF, Grimes DA. Case-control studies: research in reverse. Lancet. 2002;359(9304):431-434. doi:10.1016/S0140-6736(02)07571-2 


\section{Publish your work in this journal}

Diabetes, Metabolic Syndrome and Obesity: Targets and Therapy is an international, peer-reviewed open-access journal committed to the rapid publication of the latest laboratory and clinical findings in the fields of diabetes, metabolic syndrome and obesity research. Original research, review, case reports, hypothesis formation, expert opinion and commentaries are all considered for publication. The manuscript management system is completely online and includes a very quick and fair peer-review system, which is all easy to use. Visit http://www.dovepress.com/testimonials.php to read real quotes from published authors.

Submit your manuscript here: https://www.dovepress.com/diabetes-metabolic-syndrome-and-obesity-targets-and-therapy-journal 